\title{
Quantification of myocardial strain in patients with isolated left ventricular non- compaction and healthy subjects using deformable registration algorithm: comparison with feature tracking
}

Jia Liu ${ }^{1,2}$, Yumin $\mathrm{Li}^{1,2}$, Yue Cui ${ }^{1,2}$, Yukun Cao ${ }^{1,2}$, Sheng Yao ${ }^{3}$, Xiaoyue Zhou ${ }^{4}$, Jens Wetzl ${ }^{5}$, Wenjuan Zeng ${ }^{6^{*}}$ and Heshui Shi $^{1,2^{*}}$

\begin{abstract}
Background: Systolic dysfunction of the left ventricle is frequently associated with isolated left ventricular noncompaction (iLVNC). Clinically, the ejection fraction (EF) is the primary index of cardiac function. However, changes of EF usually occur later in the disease course. Feature tracking (FT) and deformable registration algorithm (DRA) have become appealing techniques for myocardial strain assessment.

Methods: Thirty patients with iLVNC ( $36.7 \pm 13.3$ years old) and fifty healthy volunteers ( $42.3 \pm 13.6$ years old) underwent cardiovascular magnetic resonance (CMR) examination on a 1.5 T MR scanner. Strain values in the radial, circumferential, longitudinal directions were analyzed based on the short-axis and long-axis cine images using FT and DRA methods. The iLVNC patients were further divided based on the ejection fraction, into EF $\geq 50 \%$ group $(n=11)$ and $\mathrm{EF}<50 \%$ group $(n=19)$. Receiver-operating-characteristic (ROC) analysis was performed to assess the diagnostic performance of the global strain values. Intraclass correlation coefficient (ICC) analysis was used to evaluate the intra- and inter-observer agreement.

Results: Global radial strain (GRS) was statistically lower in EF $\geq 50 \%$ group compared with control group [GRS (DRA)/\% vs. controls: $34.6 \pm 7.0$ vs. $37.6 \pm 7.2, P<0.001 ;$ GRS (FT)/\% vs. controls: $37.4 \pm 13.2$ vs. $56.9 \pm 16.4, P<0.01]$. ROC analysis of global strain values derived from DRA and FT demonstrated high area under curve (range, 0.7430.854). DRA showed excellent intra- and inter-observer agreement of global strain in both iLVNC patients (ICC: 0.995-0.999) and normal controls (ICC: 0.934-0.996). While for FT analysis, global radial strain of normal controls showed moderate intra-observer (ICC: 0.509) and poor inter-observer agreement (ICC: 0.394).

(Continued on next page)
\end{abstract}

\footnotetext{
*Correspondence: 1124177514@qq.com; heshuishi@hust.edu.cn Jia Liu and Yumin Li are the first authors of this article.

${ }^{6}$ Clinical Laboratory, Union Hospital, Tongji Medical College, Huazhong

University of Science and Technology, Wuhan 430022, China

'Department of Radiology, Union Hospital, Tongji Medical College,

Huazhong University of Science and Technology, Wuhan 430022, China

Full list of author information is available at the end of the article
}

(C) The Author(s). 2020 Open Access This article is licensed under a Creative Commons Attribution 4.0 International License, which permits use, sharing, adaptation, distribution and reproduction in any medium or format, as long as you give appropriate credit to the original author(s) and the source, provide a link to the Creative Commons licence, and indicate if changes were made. The images or other third party material in this article are included in the article's Creative Commons licence, unless indicated otherwise in a credit line to the material. If material is not included in the article's Creative Commons licence and your intended use is not permitted by statutory regulation or exceeds the permitted use, you will need to obtain permission directly from the copyright holder. To view a copy of this licence, visit http://creativecommons.org/licenses/by/4.0/ The Creative Commons Public Domain Dedication waiver (http://creativecommons.org/publicdomain/zero/1.0/) applies to the data made available in this article, unless otherwise stated in a credit line to the data. 
(Continued from previous page)

Conclusions: In patients with iLVNC, DRA can be used to quantitatively analyze the strain of left ventricle, with global radial strain being an earlier marker of LV systolic dysfunction. DRA has better reproducibility in evaluating both the global and segmental strain.

Keywords: Isolated left ventricular non-compaction, Cardiac magnetic resonance, Myocardial strain, Deformable registration algorithm, Feature tracking, Ejection fraction, Reproducibility

\section{Background}

Left ventricular non-compaction (LVNC) is an uncommon cardiomyopathy characterized by excessively prominent trabeculations and deep inter-trabecular recesses. The thickened wall of left ventricle (LV) consists of a thin compacted epicardial layer and a thick noncompacted endocardial layer. This disease is likely to result from an arrest of normal embryogenesis, mainly involving the endocardium and myocardium [1]. It may develop in isolation (isolated left ventricular noncompaction, iLVNC) or accompanied by congenital heart disease [2].

Cardiac magnetic resonance (CMR) imaging is considered as the standard of reference to clinically assess volumes and global systolic function of left ventricle [2]. LV systolic dysfunction is frequently associated with iLVNC [3]. Clinically, left ventricular ejection fraction (LVEF) is the most commonly used indicator for evaluating cardiac function. However, changes in the LVEF usually occur late in the disease course [4]. Besides, the wall motion is usually evaluated on a subjective basis and the accuracy may depend on the experience of the rater. Previous studies have shown that myocardial strain may facilitate early detection and outcome assessment of some cardiac diseases $[5,6]$. The term "strain" is defined as the change in fiber length divided by the original length, and can be quantified in three directions of myocardial fiber (radial, circumferential, and longitudinal) [7]. Several postprocessing methods have been applied using standard steady state free precession (SSFP) cine images, such as deformable registration algorithm (DRA) and feature tracking (FT). DRA and FT can evaluate both global and regional myocardial function in volunteers and patients $[8,9]$. Recent FT studies indicated that global strain values based on CMR were impaired in LVNC patients $[10,11]$. Up to date, DRA technique has not been applied to quantify myocardial strain in patients with iLVNC.

In the present study, we aimed to 1) investigate the role of strain assessment in detection of early dysfunction of left ventricle in patients with iLVNC, 2) assess the accuracy of global strain to distinguish iLVNC from healthy people, and 3) compare the reproducibility of global and segmental strains derived from DRA and FT in healthy volunteers and iLVNC patients.

\section{Methods \\ Study population}

The diagnosis of LVNC was based on the presence of established CMR and clinical criteria: 1) a thin, compacted $(\mathrm{C})$ epicardial layer and a thick, non-compacted (NC) endocardial layer with prominent trabeculations and deep intertrabecular recesses; 2) $\mathrm{NC} / \mathrm{C}$ ratio measured at end-diastole $>2.3$ in at least one LV segment [12]. Exclusion criteria included: age $<18$ years, significant valvular heart disease, clinical or CMR evidence of ischemic heart disease and evidence of concomitant congenital heart disease. Fifty age- and sex-matched healthy volunteers who responded to advertisements were recruited to participate in this study. Eventually, thirty patients with iLVNC (63.3\% males, $36.7 \pm 13.3$ years old) and fifty healthy volunteers (58\% males, $42.3 \pm 13.6$ years old) were included.

\section{CMR imaging acquisition}

All subjects underwent a standard CMR examination with a $1.5 \mathrm{~T}$ scanner (MAGNETOM Aera, Siemens Healthcare, Erlangen, Germany). A stack of short-axis views and three long-axis (2-, 3-, and 4-chamber) views were acquired. The whole LV was imaged from the base to the apex with the following parameters: repetition time (TR)/echo time (TE) of 2.9/1.2 ms, slice thickness of $6 \mathrm{~mm}$, matrix of $186 \times 256$ pixels, and flip angle of $80^{\circ}$.

\section{CMR image analysis Cardiac function}

A dedicated workstation employing commercial software (Argus, Siemens Healthcare, Erlangen, Germany) was used to analyze LV volume and function. Cardiac volumetric and functional parameters were quantified based on manual delineation of the endocardial and epicardial borders using a stack of continuous shortaxis cine images. The trabeculae and papillary muscles were included in the left ventricular cavity. The end-diastolic volume (EDV), end-systolic volume (ESV), stroke volume (SV), ejection fraction (EF) were measured and indexed to the patient's body surface area (BSA). 


\section{Strain}

Cine bSSFP-based peak systolic strains were analyzed using a prototype DRA software (TrufiStrain, version 2.0, Siemens Healthcare, Erlangen, Germany) and FT software (Medis Suite, version 3.0, Medis Medical Imaging Systems, Leiden, the Netherlands). For FT analysis, we delineated the endocardial and epicardial contours manually at end-systole and adjusted them at enddiastole. For DRA analysis, the contours were drawn manually at end-diastole; then the consecutive contours on the other phases were tracked automatically through the cardiac cycle (Fig. 1). Detailed analysis with DRA has been described previously [13]. The global longitudinal strain (GLS) was calculated based on the average of the peak systolic strain values measured by the three long axis orientations. Short-axis views of the basal, midventricular, and apical levels were used to analyze radial and circumferential strain. After a reference point was set up at the anterior insertion site, segmental circumferential strain and radial strain were calculated based on the 16-segment model. Finally, the peak systolic global and segmental myocardial strains were obtained for analysis.

\section{Statistical analysis}

SPSS (version 22.0; Chicago, IL, USA) and MedCalc (version 19.0.7; Ostend, Belgium) software was used to perform the statistical analyses. The Anderson-Darling test was used to check the normality. Continuous data were expressed as the mean \pm standard deviation. Means of two continuous normally distributed variables were compared by independent samples Student's t-test. Other comparisons were performed using the MannWhitney U test. Pairwise comparisons between groups were performed using one-way ANOVA (normal distribution) or Kruskal-Wallis test with Bonferroni correction (non-normal distribution).

Receiver-operator-characteristic (ROC) analyses of global strains determined by DRA and FT were performed to assess the diagnostic capability of strain parameters in

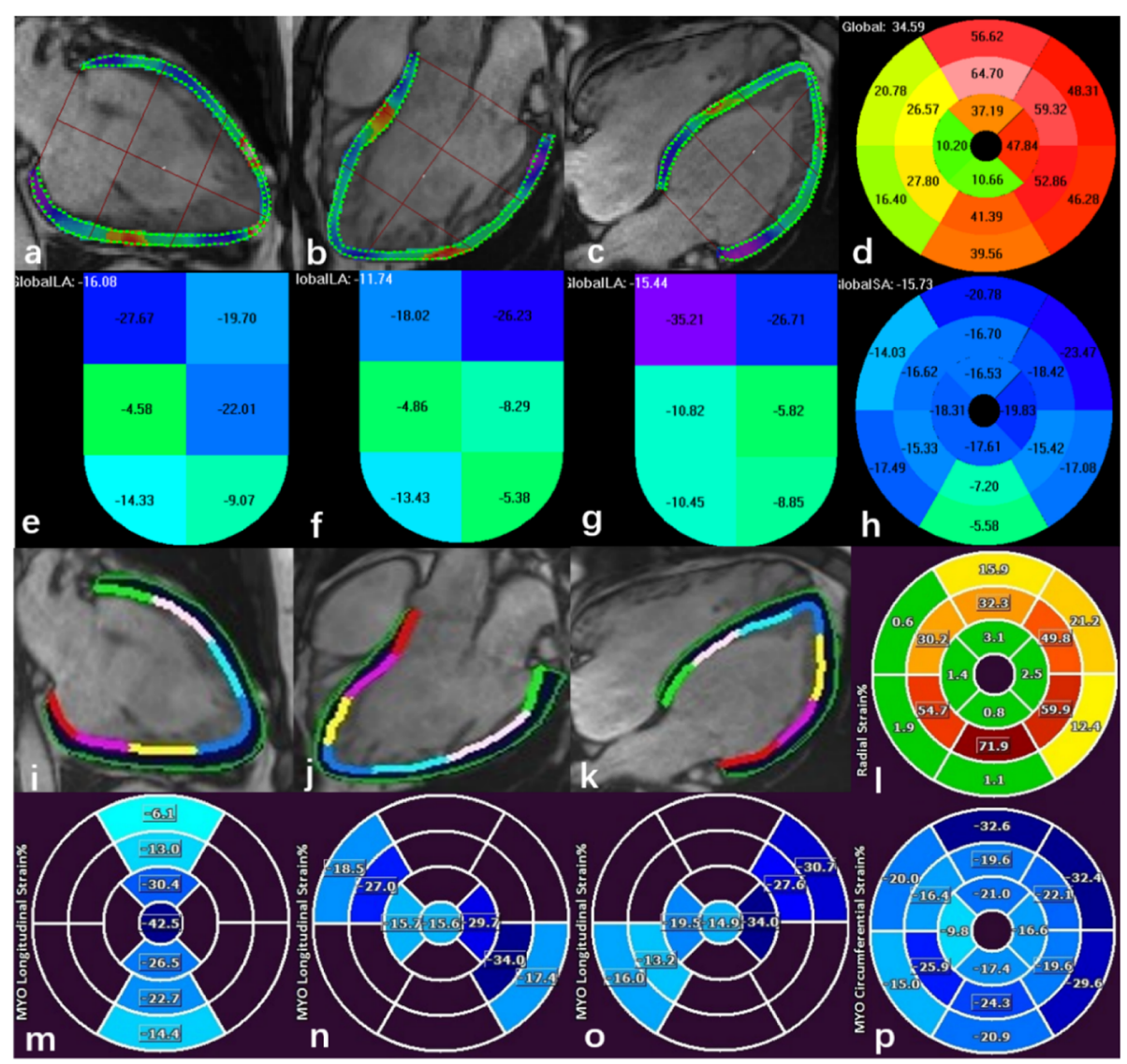

Fig. 1 Representative example of a patient with iLVNC. Measurement diagram of the peak systolic strain value of the left ventricular myocardium using DRA ( $\mathbf{a}-\mathbf{h})$ and FT (i-p) in the same patient. Images $\mathbf{a}-\mathbf{c}$, i-k show the tracking contours of longitudinal strain based on the long axis in the 2-, 3- and 4-chamber view using DRA and FT, respectively. Images e-g, $\mathbf{m}$-o show the segmental longitudinal strain values of the previous three images, respectively. Images $\mathbf{d}$ and I show the strain values of 16 segments in the radial direction using DRA and FT, respectively, which are derived from the short axis view. Images $\mathbf{h}$ and $\mathbf{p}$ show the strain values in the circumferential direction using DRA and FT, respectively, which are also derived from the short axis view 
distinguishing iLVNC from healthy volunteers. The comparisons of areas under the curve (AUC) were performed using Delong test [14]. Correlation analysis between two parameters used the Spearman rank correlation test or Pearson correlation analysis.

For strain analysis, reproducibility was tested by using the intraclass correlation coefficient (ICC) on 15 healthy volunteers and 15 iLVNC patients. Interobserver variability was analyzed on the same image set by two independent investigators (J.L., YM.L.). Intra-observer variability was analyzed on the same image set by one investigator (J.L.) with two readings 2 weeks apart. Based on the $95 \%$ confidence interval of the ICC estimate, values less than 0.50 , between 0.50 and 0.75 , between 0.75 and 0.90 , and greater than 0.90 were indicative of poor, moderate, good, and excellent reliability, respectively [15].

\section{Results}

Demographic and clinical characteristics

All demographic and cardiac MRI characteristics are shown in Table 1.

\section{Correlations between LVEF and global strain of iLVNC patients}

Correlations between left ventricular ejection fraction (LVEF) and global strain values derived from DRA and

Table 1 Clinical characteristics and parameters of cardiac function and myocardial deformation

\begin{tabular}{llll}
\hline Parameters & iLVNC $(n=30)$ & Controls $(n=50)$ & $P$ value \\
\hline Age (years) & $36.7 \pm 13.3$ & $42.3 \pm 13.6$ & 0.101 \\
Male $(\%)$ & $19(63.3 \%)$ & $29(58 \%)$ & 0.637 \\
BMI $\left(\mathrm{kg} / \mathrm{m}^{2}\right)$ & $23.2 \pm 3.7$ & $22.8 \pm 2.9$ & 0.588 \\
HR $(\mathrm{bpm})$ & $66.7 \pm 9.0$ & $65.3 \pm 9.2$ & 0.249 \\
LVEF $(\%)$ & $42.5 \pm 14.3$ & $57.7 \pm 4.7$ & $<\mathbf{0 . 0 0 1}$ \\
EDVI $\left(\mathrm{ml}^{\prime} \cdot \mathrm{m}^{-2}\right)$ & $79.5 \pm 44.8$ & $69.8 \pm 13.3$ & 0.827 \\
ESVI $\left(\mathrm{ml} \cdot \mathrm{m}^{-2}\right)$ & $49.1 \pm 40.0$ & $29.9 \pm 7.4$ & $\mathbf{0 . 0 1 6}$ \\
SVI $\left(\mathrm{ml} \cdot \mathrm{m}^{-2}\right)$ & $30.4 \pm 12.4$ & $39.9 \pm 7.4$ & $<\mathbf{0 . 0 0 1}$ \\
CI (L.min $\left.{ }^{-1} \cdot \mathrm{m}^{-2}\right)$ & $2.0 \pm 0.8$ & $2.6 \pm 0.5$ & $<\mathbf{0 . 0 0 1}$ \\
NoNC & $4.0 \pm 2.2$ & - & - \\
GLS (DRA)/\% & $-11.9 \pm 3.9$ & $-14.5 \pm 1.4$ & $\mathbf{0 . 0 0 1}$ \\
GRS (DRA)/\% & $26.3 \pm 11.0$ & $37.6 \pm 7.2$ & $<\mathbf{0 . 0 0 1}$ \\
GCS (DRA) $/ \%$ & $-12.1 \pm 4.2$ & $-16.4 \pm 1.6$ & $<\mathbf{0 . 0 0 1}$ \\
GLS (FT)/\% & $-17.8 \pm 6.4$ & $-23.3 \pm 2.8$ & $<\mathbf{0 . 0 0 1}$ \\
GRS (FT)/\% & $33.2 \pm 23.5$ & $56.9 \pm 16.4$ & $<\mathbf{0 . 0 0 1}$ \\
GCS (FT) $/ \%$ & $-15.5 \pm 6.6$ & $-22.5 \pm 2.6$ & $<\mathbf{0 . 0 0 1}$ \\
\hline BMI Body mass & &
\end{tabular}

$B M I$ Body mass index, HR Heart rate, LVEF Left ventricular ejection fraction, EDVI End-diastolic volume index, ESVI End-systolic volume index, SVI Stroke volume index, $\mathrm{Cl}$ Cardiac index, NoNC Number of non-compacted segment, DRA Deformable registered algorithm, FT Feature tracking, GRS Global radial strain, GCS Global circumferential strain, GLS Global longitudinal strain
FT in iLVNC patients are shown in Fig. 2. All global strain values showed significant correlations with LVEF $(P<0.001$ for all $)$.

\section{Comparison of strain values derived from DRA and FT among iLVNC patients with $\mathrm{EF} \geq \mathbf{5 0} \%$ group, $\mathrm{EF}<50 \%$ group, and control group}

As shown in Table 2, global radial strain (GRS) values derived from DRA and FT were significantly lower in iLVNC patients with $\mathrm{EF} \geq 50 \%$ than those in control group, whereas global circumferential strain (GCS) and global longitudinal strain (GLS) values didn't show significant difference between iLVNC patients with $\mathrm{EF} \geq$ $50 \%$ and normal subjects.

\section{Distinction of iLVNC patients and normal subjects}

The results of ROC analyses using global strains to distinguish iLVNC patients from normal controls are shown in Table 3. ROC analysis using GLS, GRS, and GCS derived from DRA and FT all demonstrated high AUC values. However, on pairwise comparison of ROC curves, there were no statistically significant differences $(P>0.05)$.

\section{Intra-observer and inter-observer reproducibility of global strains}

Intra- and inter-observer agreements of global myocardial strain for DRA and FT are shown in Table 4. DRA showed excellent intra- and inter-observer agreement in both iLVNC patients and normal controls. While for FT analysis, GRS of normal controls showed moderate intra-observer and poor inter-observer agreement. The intra- and inter-observer agreements of global strain derived from FT performed in iLVNC patients were higher than those in normal controls, especially the GRS.

\section{Intra-observer and inter-observer reproducibility of segmental strains}

As shown in Table 5, DRA showed better observer agreement for segmental strains compared with FT in both healthy volunteers and patients.

\section{Discussion}

Our study found that global radial strain derived from DRA and FT was reduced in iLVNC patients with normal EF. Global strain values had high diagnostic capability to distinguish iLVNC from healthy volunteers. Compared with FT, DRA showed better intra- and interobserver agreement for global and segmental strains in both normal controls and iLVNC patients.

In our study, global strains derived from both DRA and FT had good correlation with the LVEF. Compared to EF which acts as a global functional parameter, strain reveals the microscopic view of myocardial 

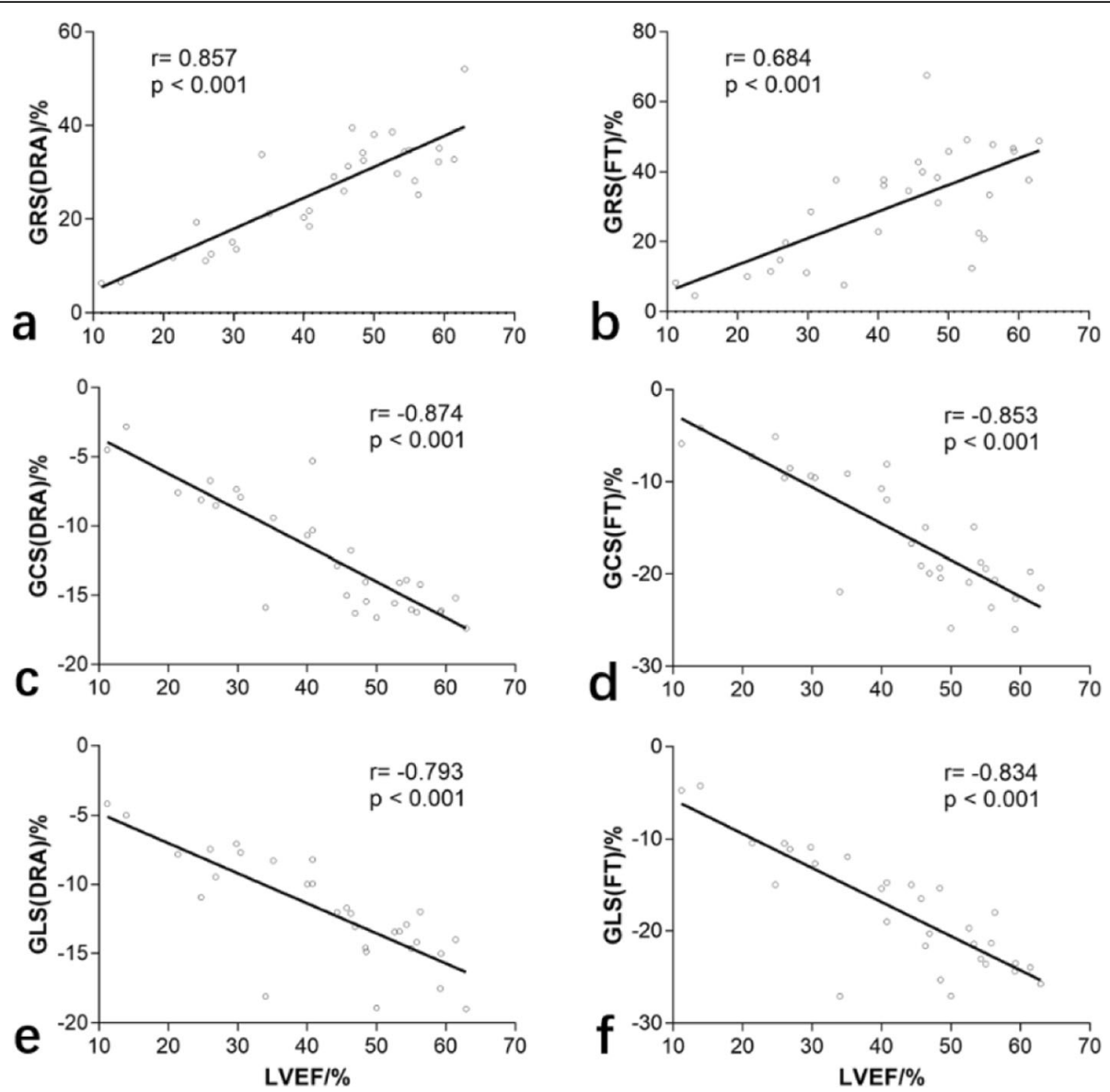

Fig. 2 Correlation between global strain values (derived from DRA and FT) and LVEF. Significant positive correlations between GRS and LVEF (image $\mathbf{a}$ and $\mathbf{b}$ ), and significant negative correlations between GCS and LVEF (image $\mathbf{c}$ and $\mathbf{d}$ ), and GLS and LVEF (image $\mathbf{e}$ and $\mathbf{f}$ ) are evident

function. Murphy and colleagues have stated that there is a long preclinical phase of disease during which patients have no symptoms or are paucisymptomatic [16]. A previous study pointed out that patients with $\mathrm{EF}<50 \%$ were eligible for drug treatment [17]. In our study, patients with iLVNC were further divided into two groups based on the ejection fraction (EF): $E F \geq 50 \%$ and $E F<50 \%$. We found that

Table 2 Comparison between iLVNC patients with EF $\geq 50 \%$, EF $<50 \%$ and normal controls

\begin{tabular}{llll}
\hline Parameters & $\mathrm{iLVNC}(n=30)$ & $\begin{array}{l}\text { Controls } \\
(n=50)\end{array}$ \\
\cline { 2 - 3 } & $\mathrm{EF} \geq 50 \%(n=11)$ & $\mathrm{EF}<50 \%(n=19)$ & $37.6 \pm 7.2$ \\
\hline GRS (DRA)/\% & $34.6 \pm 7.0^{*}$ & $21.4 \pm 10.0^{*}$ & $-16.4 \pm 1.6$ \\
GCS (DRA)/\% & $-15.6 \pm 1.1$ & $-10.0 \pm 4.0^{*}$ & $-14.5 \pm 1.4$ \\
GLS (DRA)/\% & $-14.0 \pm 2.4$ & $-10.1 \pm 3.5^{*}$ & $56.9 \pm 16.4$ \\
GRS (FT)/\% & $37.4 \pm 13.2 \#$ & $26.6 \pm 16.3^{*}$ & $-22.5 \pm 2.6$ \\
GCS (FT)/\% & $-21.3 \pm 3.2$ & $-12.2 \pm 5.7^{*}$ & $-23.3 \pm 2.8$ \\
GLS (FT)/\% & $-22.9 \pm 2.6$ & $-14.8 \pm 6.0^{*}$ & $57.7 \pm 4.7$ \\
EF & $56.4 \pm 3.9$ & $34.5 \pm 11.6^{*}$ &
\end{tabular}

$\# P<0.01$ vs. controls, ${ }^{*} P<0.001$ vs. controls
Table 3 Accuracy of the global strains (GLS, GRS and GCS) derived from two methods (DRA and FT) for the distinction of iLVNC Sensitivity (\%) Specificity (\%) AUC Cutoff value $95 \% \mathrm{Cl}$

GLS

$\begin{array}{llllll}\text { DRA } & 76.7 & 68.0 & 0.743 & -13.7 & 0.616-0.871 \\ \text { FT } & 60.0 & 100.0 & 0.794 & -18.9 & 0.680-0.908\end{array}$

GRS

\begin{tabular}{lllllll} 
DRA & 68.0 & 90.0 & & 0.850 & 35.0 & $0.765-0.935$ \\
FT & 88.0 & 73.3 & & 0.854 & 40.1 & $0.761-0.947$ \\
& \multicolumn{5}{c}{ GCS } \\
& \multicolumn{5}{c}{$0.671-0.868$} \\
DRA & 60.0 & 86.0 & 0.749 & -14.8 & 0.631 \\
FT & 70.0 & 80.0 & 0.818 & -20.0 & $0.715-0.921$
\end{tabular}

AUC Area under the curve, $\mathrm{Cl}$ Confidence interval 
Table 4 Reproducibility of the global strains (GLS, GRS and GCS) derived from two methods (DRA and FT) using intraclass correlation coefficient analysis

\begin{tabular}{|c|c|c|c|c|}
\hline & \multicolumn{2}{|c|}{ Controls } & \multicolumn{2}{|l|}{ iLVNC } \\
\hline & $\overline{D R A}$ & $\mathrm{FT}$ & DRA & $\mathrm{FT}$ \\
\hline \multicolumn{5}{|c|}{ GRS } \\
\hline inter & $0.996^{\mathrm{a}}$ & 0.394 & $0.999^{\mathrm{a}}$ & 0.716 \\
\hline intra & $0.996^{\mathrm{a}}$ & 0.509 & $0.996^{\mathrm{a}}$ & $0.785^{a}$ \\
\hline \multicolumn{5}{|c|}{ GCS } \\
\hline inter & $0.934^{a}$ & $0.891^{a}$ & $0.996^{\mathrm{a}}$ & $0.986^{\mathrm{a}}$ \\
\hline intra & $0.958^{\mathrm{a}}$ & $0.843^{\mathrm{a}}$ & $0.995^{a}$ & $0.982^{a}$ \\
\hline \multicolumn{5}{|c|}{ GLS } \\
\hline inter & $0.982^{\mathrm{a}}$ & $0.891^{\mathrm{a}}$ & $0.995^{\mathrm{a}}$ & $0.976^{\mathrm{a}}$ \\
\hline intra & $0.977^{\mathrm{a}}$ & $0.879^{a}$ & $0.997^{\mathrm{a}}$ & $0.980^{\mathrm{a}}$ \\
\hline
\end{tabular}

intra Intra-observer agreement, inter Inter-observer agreement

${ }^{a}$ Intraclass correlation coefficient $>75 \%$

GRS values derived from DRA and FT were significantly reduced in iLVNC patients with $\mathrm{EF} \geq 50 \%$. This indicates that GRS may detect early left ventricular dysfunction in iLVNC patients with normal EF. As previous studies have proposed, the compaction progress of the myocardium develops from the epicardium to the endocardium and from the ventricular base to the apex; therefore, arrest of the compaction process is prone to occur in the apical endocardium of the ventricle $[18,19]$. The left ventricular apex has a great component of radially and circumferentially oriented myocardial fibers, rather than longitudinally oriented fibers, while circumferential myofibers typically distribute in the epicardial region of LV wall [20]. The differences of anatomy may help understand the potentially superior clinical significance of radial strain in iLVNC patients.

In our study, all three global strains by the DRA and FT had high AUC values (0.743 and higher) in distinguishing iLVNC from healthy volunteers. However, there was no statistically significance difference of the AUC values between the two methods in any orientation. As mentioned, arrest of the compaction process is prone to occur in the apical endocardium of the ventricle [18]. However, in real situation, the distribution of the trabeculae may be random and complex. In addition, the strain reduction may further aggravate in the range and extent as the disease progresses, hence the global strains may be affected in all directions with no significant differences.

When evaluating the robustness of a strain-analysis method, reproducibility is an important factor. In our study, the reproducibility analysis was performed in both iLVNC patients and volunteers. We found that the intra- and inter-observer agreements of global and segmental strains were better for DRA than FT in both normal controls and iLVNC patients, especially for radial strain analysis. This is probably because that DRA is a novel method to analyze cardiac strain on a pixel-by-pixel basis. Furthermore, DRA can automatically segment the left ventricle on CMR cine images. It helps obtain the deformation from any frame to any other frame in the cardiac cycle by recovering a set of both forward and backward deformation fields. Different segmentation contours can be formed on each phase in the cardiac cycle, and then propagated to other phases $[21,22]$. Thereafter, the best combination of contour series is retained which makes the algorithm more efficient and time-saving. Although the FT algorithm has been developed for analyzing myocardial motion for a long time, applying it to clinical image data is challenging. After delineating the contours on one reliable image, the traced contours are propagated in time by identifying the same features in the following frames (optical flow technique). But some features may fade from one frame to another during tracking, and blood motion can interfere with the deformation computation [8]. It is very likely that small differences in manual contouring of endo- and epicardial borders between observers may have an important impact on radial strain measurements, while the impact on the reproducibility of longitudinal or circumferential strain is much smaller. A previous study also reported that reproducibility in DRA analysis was better than FT [23]. As mentioned above, the tracking signal sources are different between the two techniques, and readers need to adjust the contours when performing FT analysis, which is not needed when using DRA. The better reproducibility of DRA in both global and segmental view might be, more likely, related to the fully automated segmentation approach, as compared with FT [21]. A recent study comparing DRA and tagged CMR showed that the DRA demonstrated a strong relationship with the harmonic phase (HARP) for myocardial GLS $\left(R^{2}=0.75 ; P<0.0001\right)$ and GCS $\left(R^{2}=0.61 ; P<0.0001\right)$, and DRA demonstrated consistently lower coefficient of variation (CV) than tagged CMR [24].

A study using speckle tracking echocardiography indicated that apical circumferential strain may be a potential diagnostic tool for children with noncompaction cardiomyopathy (sensitivity: $87 \%$, specificity: $79 \%$, AUC: 0.88 , $P<0.001$ ) [25]. Since DRA showed better reproducibility in segmental strain in our study, we believe that it may be a good tool for evaluating the value of CMR-based segmental strain in diagnosing iLVNC patients in the future. 
Table 5 Reproducibility of segmental strains (RS, CS and LS) using two methods (DRA and FT) in controls and iLVNC patients

\begin{tabular}{|c|c|c|c|c|c|c|c|c|c|c|c|c|c|}
\hline \multirow[t]{3}{*}{ Segment } & & \multicolumn{6}{|c|}{ Controls } & \multicolumn{6}{|l|}{ iLVNC } \\
\hline & & \multicolumn{3}{|l|}{ DRA } & \multicolumn{3}{|l|}{$\mathrm{FT}$} & \multicolumn{3}{|l|}{ DRA } & \multicolumn{3}{|l|}{$\mathrm{FT}$} \\
\hline & & RS & $\mathrm{CS}$ & LS & RS & CS & LS & RS & CS & LS & RS & CS & LS \\
\hline \multirow[t]{2}{*}{1} & inter & $0.984^{\mathrm{a}}$ & $0.866^{a}$ & 0.714 & 0.266 & 0.747 & 0.702 & 0.720 & 0.704 & $0.951^{a}$ & 0.312 & 0.704 & $0.886^{a}$ \\
\hline & intra & $0.984^{\mathrm{a}}$ & $0.919^{a}$ & $0.991^{a}$ & 0.629 & $0.782^{a}$ & 0.744 & $0.978^{a}$ & $0.959^{a}$ & $0.939^{a}$ & 0.265 & 0.578 & $0.919^{a}$ \\
\hline \multirow[t]{2}{*}{2} & inter & 0.713 & $0.870^{a}$ & $0.989^{a}$ & 0.448 & 0.600 & $0.804^{a}$ & $0.979^{a}$ & 0.746 & $0.993^{a}$ & 0.561 & 0.746 & 0.532 \\
\hline & intra & $0.804^{\mathrm{a}}$ & $0.902^{\mathrm{a}}$ & $0.996^{\mathrm{a}}$ & $0.752^{\mathrm{a}}$ & 0.661 & $0.820^{\mathrm{a}}$ & $0.936^{\mathrm{a}}$ & $0.794^{\mathrm{a}}$ & $0.996^{\mathrm{a}}$ & 0.233 & 0.649 & $0.891^{a}$ \\
\hline \multirow[t]{2}{*}{3} & inter & $0.984^{\mathrm{a}}$ & $0.933^{\mathrm{a}}$ & $0.922^{\mathrm{a}}$ & 0.709 & 0.612 & $0.771^{a}$ & 0.730 & $0.823^{a}$ & $0.969^{a}$ & 0.619 & $0.823^{a}$ & $0.853^{a}$ \\
\hline & intra & $0.989^{\mathrm{a}}$ & $0.989^{a}$ & $0.936^{a}$ & $0.802^{\mathrm{a}}$ & $0.766^{a}$ & $0.806^{a}$ & $0.932^{a}$ & $0.962^{a}$ & $0.984^{a}$ & 0.618 & $0.821^{a}$ & 0.745 \\
\hline \multirow[t]{2}{*}{4} & inter & 0.706 & $0.909^{a}$ & $0.940^{a}$ & 0.369 & $0.786^{\mathrm{a}}$ & 0.578 & $0.912^{a}$ & $0.860^{a}$ & $0.979^{a}$ & 0.718 & $0.860^{a}$ & $0.946^{\mathrm{a}}$ \\
\hline & intra & 0.740 & $0.951^{a}$ & $0.971^{a}$ & 0.683 & $0.904^{\mathrm{a}}$ & 0.694 & $0.996^{\mathrm{a}}$ & $0.989^{a}$ & $0.978^{a}$ & $0.876^{a}$ & $0.805^{\mathrm{a}}$ & $0.916^{a}$ \\
\hline \multirow[t]{2}{*}{5} & inter & 0.686 & $0.910^{\mathrm{a}}$ & $0.924^{\mathrm{a}}$ & 0.174 & $0.771^{\mathrm{a}}$ & $0.809^{a}$ & $0.913^{\mathrm{a}}$ & 0.427 & $0.970^{a}$ & $0.775^{a}$ & 0.427 & $0.923^{a}$ \\
\hline & intra & 0.739 & $0.920^{\mathrm{a}}$ & $0.958^{a}$ & 0.435 & $0.800^{a}$ & $0.841^{a}$ & $0.998^{a}$ & $0.993^{a}$ & $0.965^{a}$ & 0.503 & 0.389 & 0.560 \\
\hline \multirow[t]{2}{*}{6} & inter & $0.880^{\mathrm{a}}$ & $0.915^{\mathrm{a}}$ & $0.962^{a}$ & 0.164 & $0.861^{a}$ & $0.935^{a}$ & $0.883^{a}$ & 0.274 & $0.914^{a}$ & 0.423 & 0.274 & $0.854^{a}$ \\
\hline & intra & $0.892^{\mathrm{a}}$ & $0.922^{a}$ & $0.989^{a}$ & 0.337 & $0.893^{a}$ & $0.956^{a}$ & $0.996^{a}$ & $0.858^{a}$ & $0.982^{a}$ & 0.173 & 0.338 & 0.688 \\
\hline \multirow[t]{2}{*}{7} & inter & $0.967^{\mathrm{a}}$ & $0.956^{\mathrm{a}}$ & $0.976^{\mathrm{a}}$ & 0.337 & $0.809^{a}$ & $0.761^{a}$ & $0.951^{a}$ & $0.914^{\mathrm{a}}$ & $0.955^{\mathrm{a}}$ & 0.576 & $0.914^{\mathrm{a}}$ & $0.934^{\mathrm{a}}$ \\
\hline & intra & $0.980^{\mathrm{a}}$ & $0.979^{\mathrm{a}}$ & $0.981^{a}$ & 0.403 & $0.881^{a}$ & $0.798^{a}$ & $0.998^{\mathrm{a}}$ & $0.982^{\mathrm{a}}$ & $0.978^{a}$ & 0.491 & $0.894^{a}$ & $0.868^{a}$ \\
\hline \multirow[t]{2}{*}{8} & inter & $0.915^{a}$ & $0.827^{a}$ & $0.784^{a}$ & 0.133 & 0.554 & 0.681 & $0.929^{a}$ & $0.883^{a}$ & $0.964^{a}$ & 0.074 & $0.883^{a}$ & 0.543 \\
\hline & intra & $0.936^{\mathrm{a}}$ & $0.868^{\mathrm{a}}$ & 0.680 & 0.303 & 0.656 & $0.752^{\mathrm{a}}$ & $0.994^{\mathrm{a}}$ & 0.528 & $0.956^{a}$ & 0.428 & 0.627 & $0.854^{a}$ \\
\hline \multirow[t]{2}{*}{9} & inter & 0.740 & $0.978^{\mathrm{a}}$ & $0.980^{a}$ & 0.049 & $0.850^{a}$ & 0.558 & 0.695 & $0.979^{a}$ & $0.988^{a}$ & 0.742 & $0.979^{a}$ & $0.901^{a}$ \\
\hline & intra & 0.735 & $0.975^{a}$ & $0.976^{a}$ & 0.509 & $0.875^{a}$ & $0.776^{a}$ & $0.823^{a}$ & $0.982^{a}$ & $0.991^{a}$ & $0.756^{a}$ & $0.943^{a}$ & $0.898^{a}$ \\
\hline \multirow[t]{2}{*}{10} & inter & $0.939^{\mathrm{a}}$ & $0.774^{a}$ & $0.955^{a}$ & 0.100 & 0.643 & $0.827^{\mathrm{a}}$ & $0.948^{\mathrm{a}}$ & $0.923^{\mathrm{a}}$ & $0.981^{a}$ & 0.328 & $0.923^{\mathrm{a}}$ & $0.949^{a}$ \\
\hline & intra & $0.951^{a}$ & $0.843^{a}$ & $0.945^{a}$ & 0.098 & 0.678 & $0.850^{a}$ & $0.964^{a}$ & $0.977^{a}$ & $0.921^{a}$ & 0.475 & $0.823^{a}$ & $0.920^{\mathrm{a}}$ \\
\hline \multirow[t]{2}{*}{11} & inter & $0.839^{a}$ & $0.910^{a}$ & $0.982^{\mathrm{a}}$ & 0.292 & 0.716 & $0.856^{a}$ & $0.980^{\mathrm{a}}$ & $0.916^{a}$ & $0.978^{a}$ & 0.733 & $0.916^{a}$ & $0.956^{a}$ \\
\hline & intra & $0.842^{\mathrm{a}}$ & $0.945^{\mathrm{a}}$ & $0.980^{\mathrm{a}}$ & 0.294 & 0.718 & $0.896^{a}$ & $0.992^{a}$ & $0.985^{a}$ & $0.989^{a}$ & 0.689 & 0.711 & $0.759^{a}$ \\
\hline \multirow[t]{2}{*}{12} & inter & $0.976^{a}$ & $0.794^{\mathrm{a}}$ & $0.985^{a}$ & 0.144 & 0.580 & 0.691 & $0.934^{a}$ & $0.944^{a}$ & $0.976^{a}$ & 0.658 & $0.944^{a}$ & $0.867^{a}$ \\
\hline & intra & $0.981^{\mathrm{a}}$ & $0.867^{\mathrm{a}}$ & $0.989^{a}$ & 0.310 & 0.586 & 0.710 & $0.994^{a}$ & $0.963^{a}$ & $0.994^{a}$ & 0.708 & $0.892^{a}$ & 0.723 \\
\hline \multirow[t]{2}{*}{13} & inter & $0.980^{\mathrm{a}}$ & $0.830^{\mathrm{a}}$ & $0.991^{a}$ & 0.334 & $0.890^{\mathrm{a}}$ & $0.893^{\mathrm{a}}$ & 0.579 & $0.784^{\mathrm{a}}$ & $0.994^{\mathrm{a}}$ & 0.426 & $0.784^{a}$ & $0.908^{\mathrm{a}}$ \\
\hline & intra & $0.981^{\mathrm{a}}$ & $0.861^{\mathrm{a}}$ & $0.991^{\mathrm{a}}$ & 0.367 & $0.912^{\mathrm{a}}$ & $0.898^{\mathrm{a}}$ & $0.988^{\mathrm{a}}$ & $0.975^{\mathrm{a}}$ & $0.995^{\mathrm{a}}$ & 0.541 & $0.819^{a}$ & $0.959^{a}$ \\
\hline \multirow[t]{2}{*}{14} & inter & $0.977^{a}$ & $0.957^{\mathrm{a}}$ & $0.975^{a}$ & 0.189 & $0.909^{a}$ & 0.649 & $0.848^{a}$ & 0.660 & $0.986^{a}$ & 0.418 & 0.660 & $0.953^{a}$ \\
\hline & intra & $0.989^{\mathrm{a}}$ & $0.958^{a}$ & $0.984^{a}$ & 0.335 & $0.928^{a}$ & 0.685 & $0.995^{a}$ & 0.447 & $0.961^{a}$ & 0.563 & $0.887^{a}$ & $0.834^{a}$ \\
\hline \multirow[t]{2}{*}{15} & inter & 0.739 & $0.942^{\mathrm{a}}$ & $0.975^{\mathrm{a}}$ & 0.051 & $0.838^{\mathrm{a}}$ & $0.816^{a}$ & $0.804^{\mathrm{a}}$ & $0.931^{a}$ & $0.964^{\mathrm{a}}$ & 0.352 & $0.931^{a}$ & $0.840^{\mathrm{a}}$ \\
\hline & intra & $0.791^{a}$ & $0.951^{a}$ & $0.992^{\mathrm{a}}$ & 0.095 & $0.868^{a}$ & $0.906^{\mathrm{a}}$ & $0.984^{a}$ & $0.960^{\mathrm{a}}$ & $0.993^{\mathrm{a}}$ & 0.638 & $0.936^{\mathrm{a}}$ & 0.682 \\
\hline \multirow[t]{2}{*}{16} & inter & $0.786^{\mathrm{a}}$ & $0.796^{\mathrm{a}}$ & $0.951^{\mathrm{a}}$ & 0.008 & $0.812^{\mathrm{a}}$ & $0.885^{a}$ & $0.951^{a}$ & $0.938^{a}$ & $0.997^{a}$ & 0.426 & $0.938^{a}$ & $0.954^{a}$ \\
\hline & intra & $0.808^{\mathrm{a}}$ & $0.846^{a}$ & $0.965^{a}$ & 0.024 & $0.908^{a}$ & $0.886^{a}$ & $0.998^{a}$ & 0.325 & $0.989^{a}$ & 0.552 & $0.881^{a}$ & $0.935^{\mathrm{a}}$ \\
\hline
\end{tabular}

intra Intra-observer agreement, inter Inter-observer agreement

a Intraclass correlation coefficient $>75 \%$

Meanwhile, we found that reproducibility of FT-based GRS performed in iLVNC patients was better than that in normal controls, while FT-based GCS and GLS both showed a good reproducibility in controls and iLVNC patients. The radial strain estimate heavily depends on how well the myocardial border is detected, and large variations were observed in radial strain [26]. As for the better reproducibility in iLVNC patients in our study, we assume that patients with iLVNC have thinner myocardium and weaker myocardial motion than normal people. Therefore, to some extent, the contour can be better and more accurately tracked by readers. In terms of absolute values, DRA-derived strain values were consistently lower (more negative) compared to FT, especially for radial strain values. Previous studies have demonstrated higher absolute values by FT compared to CMR tagging [27, 28]. Results of the DRA analysis in our study are within normal ranges of reported MR tagging data. Strain quantification methods differ considerably in their registration of myocardial motion, so it is 
not surprising that some techniques generate similar values $[29,30]$ while others do not $[31,32]$.

\section{Limitations}

This study has some limitations that should be acknowledged. Patients with iLVNC were retrospectively enrolled, the limitations of which are well known. Additionally, our study was a single-center study and the sample size was relatively small. We also didn't consider the possible combination of iLVNC with myocardial fibrosis.

\section{Conclusions}

In conclusion, DRA can be used to quantitatively analyze the strain of left ventricle in patients with iLVNC, and GRS is an earlier marker than LVEF of LV systolic dysfunction. Global strains can distinguish patients with iLVNC from healthy subjects with high diagnostic accuracy. Compared with FT, DRA has an excellent reproducibility in evaluating both global and segmental strain values in iLVNC patients and healthy population. Therefore, DRA can be a reliable tool to clinically assess global and segmental myocardial function of iLVNC patients.

\begin{abstract}
Abbreviations
iLVNC: Isolated left ventricular non-compaction; DRA: Deformable registration algorithm; FT: Feature tracking; LVEF: Left ventricular ejection fraction; CMR: Cardiovascular magnetic resonance; SSFP: Standard steady state free precession; BSA: Body surface area; EDVI: Left ventricular end-diastolic volume index; ESVI: End-systolic volume index; SVI: Stroke volume index; Cl: Cardiac index; GRS: Global radial strain; GCS: Global circumferential strain; GLS: Global longitudinal strain; ROC: Receiver-operator-characteristic; ICC: Intraclass correlation coefficient; AUC: Area under curve
\end{abstract}

\section{Acknowledgements}

The authors thank the study patients and volunteers for participation and the study personnel for their invaluable contribution.

\section{Authors' contributions}

HSS and WJZ conceived and designed the framework of the study. JL, YML, $Y C$, and SY contributed to the design of the study in detail. JL, YML, and YKC contributed to the cardiac and strain analysis. JL drafted the manuscript and $X Y Z$, and WJ contributed significantly to the preparation. All the authors critically revised the manuscript and gave final approval and agree to be accountable for all aspects of the work, ensuring both its integrity and accuracy.

\section{Funding}

This study was funded by Hubei Province Key Laboratory of Molecular Imaging (02.03.2018-90) and Union Hospital, Huazhong University of Science and Technology (02.03.2019-101). The funders only provided funding and had no influence on the study design, data collection, analysis, or interpretation, the decision to publish, or preparation of the manuscript.

\section{Availability of data and materials}

The datasets used or analyzed during the current study are available from the corresponding author on reasonable request

\section{Ethics approval and consent to participate}

The study was approved by the Ethics Committee of Tongji Medical College, Huazhong University of Science and Technology. Written informed consent was obtained from all the participants.
Consent for publication

Not applicable.

\section{Competing interests}

The authors declare that they have no competing interests.

\section{Author details}

'Department of Radiology, Union Hospital, Tongji Medical College, Huazhong University of Science and Technology, Wuhan 430022, China. ${ }^{2}$ Hubei Province Key Laboratory of Molecular Imaging, Wuhan 430022, China. ${ }^{3}$ Department of Orthopedics, Union Hospital, Tongji Medical College, Huazhong University of Science and Technology, Wuhan 430022, China. ${ }^{4}$ MR Collaboration, Siemens Healthineers Ltd., Shanghai, China. ${ }^{5}$ Siemens Healthcare, Erlangen, Germany. ${ }^{6}$ Clinical Laboratory, Union Hospital, Tongji Medical College, Huazhong University of Science and Technology, Wuhan 430022, China.

Received: 25 February 2020 Accepted: 13 August 2020

Published online: 03 September 2020

\section{References}

1. Towbin JA, Lorts A, Jefferies JL. Left ventricular non-compaction cardiomyopathy. Lancet. 2015;386(9995):813-25.

2. Scatteia A, Baritussio A, Bucciarelli-Ducci C. Strain imaging using cardiac magnetic resonance. Heart Fail Rev. 2017;22(4):465-76.

3. Dellegrottaglie S, Pedrotti P, Roghi A, Pedretti S, Chiariello M, Perrone-Filardi $P$. Regional and global ventricular systolic function in isolated ventricular non-compaction. Int J Cardiol. 2012;158(3):394-9.

4. Solomon SD, Anavekar N, Skali H, McMurray JJ, Swedberg K, Yusuf S, et al. Influence of ejection fraction on cardiovascular outcomes in a broad spectrum of heart failure patients. Circulation. 2005;112(24):3738-44.

5. Nucifora G, Sree Raman K, Muser D, Shah R, Perry R, Awang Ramli KA, et al. Cardiac magnetic resonance evaluation of left ventricular functional, morphological, and structural features in children and adolescents vs. young adults with isolated left ventricular non-compaction. Int J Cardiol. 2017;246:68-73.

6. Zhang KW, French B, May Khan A, Plappert T, Fang JC, Sweitzer NK, et al. Strain improves risk prediction beyond ejection fraction in chronic systolic heart failure. J Am Heart Assoc. 2014;3(1):e550.

7. Keller EJ, Fang S, Lin K, Freed BH, Smith PM, Spottiswoode BS, et al. The consistency of myocardial strain derived from heart deformation analysis. Int J Cardiovasc Imaging. 2017;33(8):1169-77.

8. Rahman ZU, Sethi P, Murtaza G, Virk HUH, Rai A, Mahmod M, et al. Feature tracking cardiac magnetic resonance imaging: a review of a novel noninvasive cardiac imaging technique. World J Cardiol. 2017;9(4):312-9.

9. Lin K, Collins JD, Chowdhary V, Markl M, Carr JC. Heart deformation analysis for automated quantification of cardiac function and regional myocardial motion patterns: a proof of concept study in patients with cardiomyopathy and healthy subjects. Eur J Radiol. 2016;85(10):1811-7.

10. Dreisbach JG, Mathur S, Houbois CP, Oechslin E, Ross H, Hanneman K, et al. Cardiovascular magnetic resonance based diagnosis of left ventricular noncompaction cardiomyopathy: impact of cine bSSFP strain analysis. J Cardiovasc Magn Reson. 2020;22(1):9.

11. Gastl M, Gotschy A, Polacin M, Vishnevskiy V, Meyer D, Sokolska J, et al. Determinants of myocardial function characterized by CMR-derived strain parameters in left ventricular non-compaction cardiomyopathy. Sci Rep. 2019;9(1):15882.

12. Petersen SE, Selvanayagam JB, Wiesmann F, Robson MD, Francis JM, Anderson $\mathrm{RH}$, et al. Left ventricular non-compaction: insights from cardiovascular magnetic resonance imaging. J Am Coll Cardiol. 2005;46(1): $101-5$.

13. Liu H, Yang D, Wan K, Luo Y, Sun JY, Zhang TJ, et al. Distribution pattern of left-ventricular myocardial strain analyzed by a cine MRI based deformation registration algorithm in healthy Chinese volunteers. Sci Rep. 2017;7:45314.

14. Mehta CR, Patel NR, Tsiatis AA. Exact significance testing for ordered categorical data. Biometrics. 1984:40(3):819-25.

15. Koo TK, Li MY. A guideline of selecting and reporting intraclass correlation coefficients for reliability research. J Chiropr Med. 2016;15(2):155-63.

16. Murphy RT, Thaman R, Blanes JG, Ward D, Sevdalis E, Papra E, et al. Natural history and familial characteristics of isolated left ventricular noncompaction. Eur Heart J. 2005;26(2):187-92. 
17. Jessup M, Abraham WT, Casey DE, Feldman AM, Francis GS, Ganiats TG, et al. 2009 focused update: ACCF/AHA guidelines for the diagnosis and management of heart failure in adults. Circulation. 2009;1 19(14):1977-2016.

18. Sedmera D, Thomas PS. Trabeculation in the embryonic heart. Bioessays. 1996;18(7):607.

19. Mohun TJ, Weninger WJ. Imaging heart development using high-resolution episcopic microscopy. Curr Opin Genet Dev. 2011;21(5):573-8.

20. Kowalski M, Kukulski T, Jamal F, D'hooge J, Weidemann F, Rademakers F, et al. Can natural strain and strain rate quantify regional myocardial deformation? A study in healthy subjects. Ultrasound Med Biol. 2001;27(8): 1087-97.

21. Jolly MP, Guetter C, Lu X, Xue H, Guehring J. Automatic segmentation of the myocardium in cine MR images using deformable registration. In: Camara O, Konukoglu E, Pop M, Rhode K, Sermesant M, Young A, editors. Statistical atlases and computational models of the heart. Imaging and modelling challenges; 2011. p. 98-108.

22. Jolly MP, Guetter C, Guehring J. Cardiac segmentation in MR cine data using inverse consistent deformable registration. In: Proceedings of 2010 7th IEEE international symposium on biomedical imaging: from nano to macro, ISB|; 2010. p. 484-7.

23. Wang J, Li W, Sun J, Liu H, Kang Y, Yang D, et al. Improved segmental myocardial strain reproducibility using deformable registration algorithms compared with feature tracking cardiac MRI and speckle tracking echocardiography. J Magn Reson Imaging. 2018;48(2):404-14.

24. Lamacie MM, Houbois CP, Greiser A, Jolly MP, Thavendiranathan P, Wintersperger BJ. Quantification of myocardial deformation by deformable registration-based analysis of cine MRI: validation with tagged CMR. Eur Radiol. 2019;29(7):3658-68.

25. Yubbu P, Nawaytou HM, Calderon-Anyosa R, Banerjee A. Diagnostic value of myocardial deformation pattern in children with noncompaction cardiomyopathy. Int J Cardiovasc Imaging. 2018;34(10):1529-39.

26. Schuster A, Paul M, Bettencourt N, Morton G, Chiribiri A, Ishida M, et al. Cardiovascular magnetic resonance myocardial feature tracking for quantitative viability assessment in ischemic cardiomyopathy. Int J Cardiol. 2013;166(2):413-20.

27. Moody WE, Taylor RJ, Edwards NC, Chue CD, Umar F, Taylor TJ, et al. Comparison of magnetic resonance feature tracking for systolic and diastolic strain and strain rate calculation with spatial modulation of magnetization imaging analysis. J Magn Reson Imaging. 2015;41(4):1000-12.

28. Augustine D, Lewandowski AJ, Lazdam M, Rai A, Francis J, Myerson S, et al. Global and regional left ventricular myocardial deformation measures by magnetic resonance feature tracking in healthy volunteers: comparison with tagging and relevance of gender. J Cardiovasc Magn Reson. 2013;15(1):8.

29. Neizel M, Lossnitzer D, Korosoglou G, Schaufele T, Lewien A, Steen H, et al. Strain-encoded (SENC) magnetic resonance imaging to evaluate regional heterogeneity of myocardial strain in healthy volunteers: comparison with conventional tagging. J Magn Reson Imaging. 2009;29(1):99-105.

30. Hor KN, Gottliebson WM, Carson C, Wash E, Cnota J, Fleck R, et al. Comparison of magnetic resonance feature tracking for strain calculation with harmonic phase imaging analysis. JACC Cardiovasc Imaging. 2010;3(2): 144-51.

31. Zhong J, Yu X. Strain and torsion quantification in mouse hearts under dobutamine stimulation using 2D multiphase MR DENSE. Magn Reson Med. 2010;64(5):1315-22.

32. Goo-Yeong C, Jonathan C, Rodel L, Mark S, Thomas HM. Comparison of two-dimensional speckle and tissue velocity based strain and validation with harmonic phase magnetic resonance imaging. Am J Cardiol. 2006; 97(11):1661-6

\section{Publisher's Note}

Springer Nature remains neutral with regard to jurisdictional claims in published maps and institutional affiliations.

Ready to submit your research? Choose BMC and benefit from:

- fast, convenient online submission

- thorough peer review by experienced researchers in your field

- rapid publication on acceptance

- support for research data, including large and complex data types

- gold Open Access which fosters wider collaboration and increased citations

- maximum visibility for your research: over $100 \mathrm{M}$ website views per year

At BMC, research is always in progress.

Learn more biomedcentral.com/submissions 